\title{
La enseñanza de los primeros auxilios en educación física: revisión sistemática acerca de los materiales para suimplementación
}

\author{
First aid teaching in physical education: a systematic review about the materials for its implementation \\ Joaquín Lago-Ballesteros, Silvia Basanta-Camiño, Ruben Navarro-Paton \\ Universidad de Santiago de Compostela (España)
}

\begin{abstract}
Resumen. El objetivo del presente estudio consiste en realizar una revisión sistemática de la literatura científica centrándose en el análisis de los materiales utilizados en programas escolares para la formación en primeros auxilios. Se consideraron susceptibles de ser incluidos, aquellos artículos de investigación originales que contenían programas de enseñanza o intervenciones desarrolladas en el ámbito escolar, dirigidas a alumnos entre 6 y 18 años, en los que se trabajaba cualquier aspecto relacionado con los primeros auxilios. Fueron exploradas las principales bases de datos en inglés y español correspondientes a los ámbitos de la educación y la salud en sus versiones disponibles en línea en abril de 2018 . Un total de 27 artículos fueron finalmente objeto de la revisión y se procedió a la síntesis de la información que contenían. Los materiales empleados en los diferentes programas formativos fueron muy variados. La mayoría de los programas optan por una combinación de materiales didácticos generales y específicos, siendo la opción más habitual la mezcla de material audiovisual con material no fungible, móvil, complementario y de medición. En conclusión, existe una enorme variedad de materiales a disposición de los maestros en educación física para llevar a cabo la enseñanza de los primeros auxilios, por lo que, a la hora de diseñar un programa formativo eficaz, deberían emplearse criterios didácticos para seleccionar y combinar los materiales a emplear.

Palabras clave. Reanimación cardiopulmonar, soporte vital básico, emergencia, material didáctico, programas formativos.
\end{abstract}

Abstract. The aim of the present study was to undertake a systematic review with a special focus on the materials used in school-based first aid training programs. Eligible studies included school-based first aid interventions targeting students aged between 6 and 18 years. Major online databases in the areas of education and health were searched, for peer-reviewed publications available as at April 2018. A total of 27 journal articles were relevant to the review. The materials used in the different training programs showed a wide variety. Most programs combined general and specific teaching materials. The most common option was a mixture of audiovisual material with non-expendable, mobile, additional and measurement material. In conclusion, a wide variety of materials are available to physical education teachers to carry on first-aid training. Consequently, didactic criteria should be employed for material selection and combination when an effective training program is intended.

Keywords. Cardiopulmonary resuscitation, basic life support, emergency, teaching materials, training programs.

\section{Introducción}

Los cursos de formación en primeros auxilios son ofertados para el público en general en multitud de organizaciones y entidades. Hasta hace unos pocos años, cuando se pensaba en la formación en primeros auxilios, rara vez se hacía referencia a los mismos como parte del currículo educativo o de las programaciones de aula. Sin embargo, en el transcurso de las dos últimas décadas, los primeros auxilios parecen haber cobrado una mayor presencia entre los contenidos considerados en las etapas que componen la escolaridad obligatoria. Muchas entidades médicas, como la American Heart Association (AHA, 2001) y el European Resuscitation Council (ERC) (Nolan, et al., 2010) de común acuerdo con la Organización Mundial de la Salud(OMS), publicaron la recomendación para la enseñanza de la RCP a los niños llamada «Hands that help-Training children is training for life» (Böttiger, et al., 2015), aconsejando la inclusión de la enseñanza de primeros auxilios en las escuelas e institutos, haciendo referencia no solo a una educación para la salud y la prevención de enfermedades y accidentes, sino también a la actuación en caso de ser necesaria la práctica de una Reanimación Cardiopulmonar básica (RCP) (Miró, Díaz, Escalada, Pérez-Pueyo, \& Sánchez, 2013).

Esto se debe a que la escolarización es obligatoria en los países desarrollados, por lo que las escuelas y los institutos serían los lugares ideales para llegar al mayor número posible de personas, consiguiendo así iniciar casi a la totalidad de la población en el aprendizaje, conocimiento y práctica de las técnicas básicas de actuación en primeros auxilios en general, y sobre todo en la ejecución de la RCP (Miró, et al., 2013). Otras razones básicas para escoger a la población infantil y joven como receptores de esta enseñanza se deben a su carácter más asequible, su capacidad de aprender fácilmente y retener conocimientos, su interés en adquirir nuevas habilidades, son fácilmente motivables, y probablemente también más predispuestos a administrar primeros auxilios en caso de que una persona lo necesite (García-Vega, Montero-Pérez, \& Encinas-Puente, 2008; Jelinek, et al., 2001).

Fecha recepción: 15-01-18. Fecha de aceptación: 28-05-18 Joaquín Lago-Ballesteros

joaquin.lago@usc.es
Además, existen estudios con resultados positivos en el aprendizaje temprano de los primeros auxilios, concluyendo que una persona a partir de trece años tiene suficiente capacidad física para realizar una RCP y también suficiente madurez para comprender la importancia de actuar si se encuentra ante una situación de emergencia (Jones, et al., 2007).

Por otro lado, los docentes deberían siempre estar capacitados para atender cualquier situación de emergencia que pueda ocurrir en torno a los escolares, puesto que los accidentes ocurren muchas veces en la escuela, donde los niños pasan la mayor parte de su tiempo (Ríos, 2011). En concreto, la educación física es el área más propensa a que se produzcan accidentes debido a su naturaleza práctica, por lo que debería ser imprescindible que las maestras y maestros encargados de su docencia estén preparados para actuar en caso de ser necesario y que, a su vez, preparen a su alumnado para hacer lo propio (Navarro, Arufe, \& Basanta, 2015)

Analizando los Reales Decretos educativos españoles, y refiriéndonos a la Educación Primaria, en España, los contenidos sobre primeros auxilios fueron incorporados con la Ley Orgánica 8/2013, de 9 de diciembre, para la mejora de la calidad educativa (LOMCE), si bien anteriormente ya estaban presentes en la legislación educativa de algunas comunidades autónomas. En el desarrollo esta ley, por medio del Real Decreto 126/2014, de 28 de febrero, por el que se establece el currículo básico de la Educación Primaria, los primeros auxilios aparecen de manera explícita, dentro de las materias de Ciencias de la naturaleza, Valores sociales y cívicos y Educación física. En el caso de la Educación física, los primeros auxilios semencionan dentro deun estándar de aprendizaje: «Explica y reconoce las lesiones y enfermedades deportivas más comunes, así como las acciones preventivas y los primeros auxilios» (Real Decreto, 126/2014, p. 19409). Asimismo, los primeros auxilios se pueden relacionar de forma implícita con los elementos transversales de esta etapa, ya que se incorporan elementos curriculares relacionados con la protección ante emergencias y catástrofes, entre otros.

En lo que se refiere a la etapa de secundaria, el Real Decreto 1105/ 2014, de 26 de diciembre, por el que se establece el currículo básico de la Educación Secundaria Obligatoria y del Bachillerato incluye los primeros auxilios en las asignaturas de Valores éticos ( $\left.1^{\circ} \mathrm{ESO}\right)$, Iniciación a 
la Actividad Emprendedora y Empresarial ( $\left.4^{\circ} \mathrm{ESO}\right)$ y Educación física (de $1^{\circ} \mathrm{ESO}$ a $1^{\circ}$ Bachillerato). En concreto, en Educación física de primer ciclo $\left(1^{\circ}, 2^{\circ} y^{\circ} \mathrm{ESO}\right)$, se expone el siguiente criterio de evaluación, junto con sus correspondientes estándares de aprendizaje:

9. Controlar las dificultades y los riesgos durante su participación en actividades físico-deportivas y artístico-expresivas, analizando las características de las mismas y las interacciones motrices que conllevan, y adoptando medidas preventivas y de seguridad en su desarrollo. 9.1. Identifica las características de las actividades físico-deportivas y artístico-expresivas propuestas que pueden suponer un elemento de riesgo para sí mismo o para los demás. 9.2. Describe los protocolos a seguir para activar los servicios de emergencia y de protección del entorno. 9.3. Adopta las medidas preventivas y de seguridad propias de las actividades desarrolladas durante el ciclo, teniendo especial cuidado con aquellas que se realizan en un entorno no estable. (Real Decreto 105/2014, p.484)

Asimismo, en Educación física de $4^{\circ} \mathrm{ESO}$, el criterio de evaluación que hace referencia a los primeros auxilios y sus estándares de aprendizaje evaluables son:

10. Asumir la responsabilidad de la propia seguridad en la práctica de actividad física, teniendo en cuenta los factores inherentes a la actividad y previendo las consecuencias que pueden tener las actuaciones poco cuidadosas sobre la seguridad de los participantes. 10.1 . Verifica las condiciones de práctica segura usando convenientemente el equipo personal y los materiales y espacios de práctica. 10.2. Identifica las lesiones más frecuentes derivadas de la práctica de actividad física. 10.3. Describe los protocolos que deben seguirse ante las lesiones, accidentes o situaciones de emergencia más frecuentes producidas durante la práctica de actividades físico deportivas. (Real Decreto 105/ 2014, p.485)

Por último, dentro deEducación física de $1^{\circ}$ Bachillerato, se encuentra una referencia a la previsión y control de riesgos, aunque ya desaparecen los primeros auxilios de forma explícita.

De esta forma, es lógico pensar que los maestros y maestras de estas etapas educativas incluyen contenidos sobre primeros auxilios en sus programaciones de aula, sobre todo los encargados de impartir las asignaturas que los integran en el currículo educativo, entre ellos, el docente de educación física (Navarro, et al., 2015). Sin embargo, algunos estudios revelan que los conocimientos que poseen sobre la materia los actuales maestros y los futuros maestros de educación primaria son escasos e insuficientes (López-Felpeto, Navarro-Patón, \& BasantaCamiño, 2015; Mpotos, Vekeman, Monsieurs, Derese, \& Valcke, 2013; Navarro-Patón, Basanta-Camiño, Abelairas-Gómez, \& López-García, 2015; Navarro-Patón, Penelas-Teijeiro, \& Basanta-Camiño, 2016), por lo que muchos de ellos tampoco se sienten capacitados para incluir este contenido en sus aulas (Mpotos et al., 2013).

Por otro lado, a la hora de impartir contenidos en la escuela sobre primeros auxilios, se requiere la creación de programas diseñados de forma específica para ello, atendiendo a todos los aspectos pedagógicos clave para su éxito (Miró, Díaz, Escalada, Pérez-Pueyo, \& Sánchez, 2012; Miró, et al., 2013). Para obtener buenos resultados, intervienen muchos factores, que van a convertirse en aspectos primordiales a tener en cuenta para su puesta en marcha y para el mantenimiento de estos programas. Uno de ellos es el material necesario (Miró, et al., 2012; Miró, et al., 2013). En la mayoría de las investigaciones y estudios realizados, el material empleado ocupa una parte importante del programa, siendo considerado incluso como condicionante de la eficacia en la transmisión de conocimientos y promoción de habilidades (NavarroPatón, et al., 2018).

La falta de fondos y, por tanto, de material, es una de las preocupaciones de los docentes a la hora de abordar los primeros auxilios con sus estudiantes, llegando en ocasiones a manifestar que no se imparten estos contenidos curriculares debido a que se carece del tiempo, de los fondos o del material necesarios para implementarlos (Reder \& Quan, 2003). Sin duda, como ocurre con cualquier otro contenido, la implementación de contenidos sobre primeros auxilios en el aula requie- re de métodos y recursos adecuados, pues estos van a influir en los aprendizajes posteriores (Navarro-Patón, et al., 2015). Una de las posibilidades para lograr recursos óptimos es buscar la colaboración con empresas privadas, que pondrían a disposición de los centros implicados el material adecuado para este tipo de enseñanzas (Liberman, Golberg, Mulder, \& Sampalis, 2000), pero esta opción no siempre es viable.

Aunque no siempre se dispone del material y recursos deseables, conforme a los programas educativos existentes recomendados por expertos (López-Felpeto, et al., 2015), dependerá del interés, dedicación e ingenio del docente, la búsqueda de alternativas que permitan tratar los contenidos programados. Cabe destacar que no se trataría de reducir la formación en primeros auxilios a clases magistrales, sino de experimentar elementos innovadores y tecnológicos que permitan influir de manera significativa en los educandos, en este caso, para «conseguir una construcción de saberes y procedimientos que permitan activar la cadena de supervivencia, elemento esencial para salvar vidas» (Teijeiro-Blanco, Navarro-Patón, \& Basanta-Camiño, 2017, p.116).

En definitiva, con intención de ayudar a todo docente que desee indagar estrategias y métodos de instrucción que permitan un buen aprendizaje, el objetivo del presente estudio consiste en realizar una revisión sistemática de la literatura científica existente para analizar los materiales utilizados en programas escolares para la formación en primeros auxilios.

\section{Material y método}

\section{Criterios de inclusión}

A la hora de juzgar si un estudio debía ser tenido en cuenta para su posterior análisis, se consideraron susceptibles de ser incluidos a aquellos artículos de investigación originales que contenían programas de enseñanza o intervenciones desarrolladas en el ámbito escolar, dirigidas a alumnos entre 6 y 18 años, en los que se trabajaba cualquier aspecto relacionado con los primeros auxilios. No se establecieron limitaciones ni en cuanto al tipo de diseño empleado ni respecto al tipo de objetivos planteados en la intervención que se recoge en cada uno de los estudios (i.e. conceptuales, procedimentales y actitudinales). Además de estos criterios, se establecieron restricciones en cuanto al idioma, considerando para su inclusión sólo a aquellos redactados en inglés o español, y al tipo de documento, descartando informes secundarios, editoriales, ensayos y artículos de opinión.

\section{Fuentes de datos y ecuaciones de búsqueda}

La revisión fue realizada a partir de una búsqueda de artículos de revista en las principales bases de datos en inglés y español correspondientes a los ámbitos de la educación y la salud. En concreto, las bases de datos exploradas fueron PsycINFO, ERIC, Proquest Education and Psychology, PubMed, IBECS, LILACS, Scielo España, y Dialnet. Estas bases de datos fueron exploradas en sus versiones disponibles en línea en abril de 2018. El proceso de búsqueda se repitió en cada una de las bases de datos empleando las ecuaciones de búsqueda que se presentan en la Tabla 1. Para lograr un proceso más exhaustivo, tras la identificación de los resultados relevantes se procedió a revisar las referencias bibliográficas, tanto de los artículos de investigación originales como de las revisiones de literatura, tratando de identificar trabajos originales que pudieran haber quedado fuera del espectro de la búsqueda.

Tabla 1

Ecuaciones de búsqueda a partir de las palabras clave

Ecuación de búsqueda en ingles

school OR educat* ${ }^{*}$ OR student OR chil* OR adolescen* OR teen* OR youth

AND

first aid OR cardiopulmonary resuscitation OR CPR OR automated external defibrillation OR AED OR basic life support OR emergency treatment OR emergency response OR life saving skills OR bystander trauma care OR bystander response

AND

interven* OR program* OR train* OR teach* OR learn* OR evaluat* OR prevent* Ecuación de búsqueda en español

escuela OR escol* OR educa* OR alumn* OR niñ* OR adolescen* OR joven*

AND

primeros auxilios OR reanimación cardiopulmonar OR resucitación cardiopulmonar OR RCP OR desfibrilación externa automática OR DEA OR soporte vital básico OR emergencia* AND interven* OR program* OR enseñanza OR formación OR aprend* OR preven* 


\section{Síntesis de la información}

Tras completar la búsqueda y depurar los resultados, eliminando aquellos trabajos que pudieran aparecer duplicados o que no constituyesen artículos de investigación originales, el título y el resumen de cada uno de los resultados obtenidos en el proceso de búsqueda fue examinado para evaluar el cumplimiento de los criterios de inclusión. En aquellos casos en los que la información proporcionada en dichos apartados resultó insuficiente para determinar la inclusión o exclusión del estudio, se procedió al análisis del texto completo. Todo este proceso fue realizado por dos de los autores, empleando el juicio del tercer autor para resolver las escasas discrepancias apreciadas. Tras este filtrado, un total de 27 artículos fueron considerados relevantes para los fines de la presente revisión y se procedió a la síntesis de la información contenida en los mismos. Con el fin de superar el análisis individual o anecdótico de cada elemento material mencionado en los distintos programas, se procedió a agruparlos en función de sus características didácticas más relevantes, para lo que se empleó la clasificación de materiales didácticos en educación física de Fernández (1999).

\section{Resultados}

La estrategia de búsqueda descrita arrojó, inicialmente, un total de 331 artículos susceptibles de ser incluidos en la presente revisión. Pero este amplio número conllevó que la mayor parte de ellos debiese ser excluido, al carecer de relevancia, conforme a los criterios de inclusión anteriormente establecidos. De esta forma, 288 artículos fueron exclui- dos por abordar los primeros auxilios desde una perspectiva distinta a la escogida en este trabajo, esto es, no contenían programas de enseñanza o intervenciones desarrolladas en el ámbito escolar. Asimismo, otros 16 resultados fueron excluidos por diversas razones: a pesar de incluir un programa formativo llevado a cabo en el entorno escolar, este no se dirigía a alumnos de entre 6 y 18 años, sino a la etapa de infantil ( $\mathrm{N}=3)$, programas piloto cuya versión mayor fue incluida $(\mathrm{N}=4)$, el texto completo estaba redactado en un idioma distinto del inglés o español ( $N$ = 5), no proporcionaban suficiente información sobre los materiales utilizados ( $\mathrm{N}=2$ ) o, las muestras eran demasiado pequeñas $(\mathrm{N}=2)$.

Tras el filtrado de los resultados, 27 artículos fueron finalmente objeto de la revisión y se procedió a la síntesis de la información que contenían. De entre ellos, 7 incluyeron programas de enseñanza dirigidos a la etapa de educación primaria (Frederick, Bixby, Orzel, StewartBrown, \& Willett, 2000; Lester, Donnelly, Weston, \& Morgan, 1996; Louis, et al., 2015; Lubrano, et al., 2005; Marchand-Martella, Martella, Christensen, Agran, \& Young, 1992; Palacio, Nonide, Carreño, López, \& Cao, 2015; Toner, et al., 2007), 16 dirigieron su intervención a la etapa de educación secundaria (Carruth, et al., 2010; Cerezo Espinosa, et al., 2018; Engeland, Roysamb, Smedslund, \& Sogaard, 2002; Garcia del Aguila, et al., 2014; Iserbyt, Charlier, \& Mols, 2014; Jimenez-Fabrega, et al., 2009; Kelley, et al., 2006; Lester, Donnelly, \& Weston, 1997; López, Freire, Rasines, \& Iglesias, 2012; Marchiori et al., 2012; Meissner, Kloppe, \& Hanefeld, 2012; Onyeaso, 2016; Reder, Cummings, \& Quan, 2006; Van Raemdonck, Monsieurs, Aerenhouts, \& De Martelaer, 2014; Van Raemdonck, Aerenhouts, Monsieurs, \& De Martelaer, 2017;

Tabla 2

Materiales didácticos generales empleados en los distintos programas formativos (taxonomía de Fernández, 1999)

\begin{tabular}{|c|c|c|c|c|c|}
\hline Tipo de material & Elemento material & Nombre de los programas & Contenidos & Referencias & $\begin{array}{c}\text { Etapa } \\
\text { educativa }\end{array}$ \\
\hline \multirow{6}{*}{ Material impreso } & Manual o libro de texto & $\begin{array}{l}\text { - Injury Minimization Programme for Schools } \\
\text { - Mouse Calls } \\
\text { - Do Something } \\
\text { - F.A.R.M.E. } \\
\text { - Becoming an under } 11 \text { rescuer } \\
\text { ABC for life }\end{array}$ & $\begin{array}{l}\text { - Prevención de accidentes } \\
\text { - } \text { SVB } \\
\text { RCP } \\
\text { Primeros auxilios ante diferentes tipos de accidentes } \\
\text { y/o lesiones }\end{array}$ & $\begin{array}{l}\text { (Carruth et al., 2010; Engeland et al., } \\
\text { 2002; Frederick et al., 2000; Lubrano } \\
\text { et al., 2005; Marchand-Martella et al., } \\
\text { 1992; Toner et al., 2007) }\end{array}$ & $\begin{array}{l}\text { Primaria } \\
\text { Secundaria }\end{array}$ \\
\hline & Unidades Didácticas & $\begin{array}{ll} & \text { Longfellow's WHALE Tales K-6 (American } \\
\text { Red Cross, 2009) } \\
\text { - RCP na aula } \\
\text { - Becoming an under } 11 \text { rescuer } \\
\text { - OSNR }\end{array}$ & $\begin{array}{ll} & \text { Seguridad en el agua } \\
\text { - SVB pediátrico } \\
\text { - } & \text { DCP } \\
\text { - } & \text { PESA } \\
& \text { roto) } \\
\end{array}$ & $\begin{array}{l}\text { (López et al., 2012; Lubrano et al., } \\
\text { 2005; Turgut et al., 2016; Villanueva } \\
\text { et al., 2018) }\end{array}$ & $\begin{array}{l}\text { Primaria } \\
\text { Secundaria }\end{array}$ \\
\hline & Fichas descriptivas & $\begin{array}{l}\text { - Longfellow's WHALE Tales K-6 (American } \\
\text { Red Cross, 2009) }\end{array}$ & - Seguridad en el agua & (Turgut et al., 2016) & $\begin{array}{l}\text { Primaria } \\
\text { Secundaria }\end{array}$ \\
\hline & Fichas de actividades & $\begin{array}{l}\text { - Longfellow's WHALE Tales K-6 (American } \\
\text { Red Cross, 2009) } \\
\text { - OSNR } \\
\end{array}$ & $\begin{array}{l}\text { - Seguridad en el agua } \\
\text { - RCP }\end{array}$ & $\begin{array}{l}\text { (Lester et al., 1996; Turgut et al., } \\
\text { 2016; Villanueva et al., 2018) }\end{array}$ & $\begin{array}{l}\text { Primaria } \\
\text { Secundaria }\end{array}$ \\
\hline & Diploma & $\begin{array}{l}\text { Becoming an under } 11 \text { rescuer } \\
\text { - ABC for life }\end{array}$ & $\begin{array}{ll} & \text { SVB } \\
\text { - } & \text { RCP } \\
\text { Primeros auxilios ante hemorragia nasal y diente roto } \\
\end{array}$ & $\begin{array}{l}\text { (Lubrano et al., 2005; Toner et al., } \\
\text { 2007) }\end{array}$ & Primaria \\
\hline & Cuento & - Con tus manos puedes salvar vidas & · SVB & (Palacio et al., 2015) & Primaria \\
\hline \multirow{3}{*}{$\begin{array}{l}\text { Material visual fijo } \\
\text { no proyectable }\end{array}$} & Carteles & $\begin{array}{l}\text { - Longfellow's WHALE Tales K-6 (American } \\
\text { Red Cross, 2009) } \\
\text { - ABC for life } \\
\end{array}$ & $\begin{array}{ll}\text { - } & \text { Seguridad en el agua } \\
\text { - } & \text { RVB } \\
\end{array}$ & (Toner et al., 2007; Turgut et al., 2016) & $\begin{array}{l}\text { Primaria } \\
\text { Secundaria }\end{array}$ \\
\hline & Pentadíptico desplegable & - Cardiomaratón & $\begin{array}{ll} & \text { Reconocer PCR } \\
\text { - } & \text { RCP } \\
\text { - PLS } & \text { cadena de supervivencia } \\
\text { - } & \text { Llamada a centro coordinador de urgencias } \\
\text { - Atragantamiento y desobstrucción de vía aérea }\end{array}$ & (Garcia del Aguila et al., 2014) & Secundaria \\
\hline & Insignia & - Becoming an under 11 rescuer & $\begin{array}{l}\text { - Primeros auxilios ante hemorragia nasal y diente roto } \\
\text { - SVB pediátrico }\end{array}$ & (Lubrano et al., 2005) & Primaria \\
\hline $\begin{array}{l}\text { Material visual fijo } \\
\text { proyectable }\end{array}$ & Presentaciones en PPT & $\begin{array}{l}\text { - Con tus manos puedes salvar vidas } \\
\text { OSNR }\end{array}$ & $\begin{array}{ll}\text { - } & \text { SVB } \\
\text { - } & \text { RCP }\end{array}$ & $\begin{array}{l}\text { (Bohn et al., 2012; Cerezo Espinosa et } \\
\text { al., 2018; Onyeaso, 2016; Palacio et } \\
\text { al., 2015; Villanueva et al., 2018) }\end{array}$ & $\begin{array}{l}\mathrm{t} \text { Primaria } \\
\text { Secundaria }\end{array}$ \\
\hline Material auditivo & Canción & $\begin{array}{l}\text { - Con tus manos puedes salvar vidas } \\
\text { - OSNR }\end{array}$ & $\begin{array}{ll}\text { - } & \text { SVB } \\
\text { - } & \text { RCP }\end{array}$ & (Onyeaso, 2016; Palacio et al., 2015) & $\begin{array}{l}\text { Primaria } \\
\text { Secundaria }\end{array}$ \\
\hline Material audiovisual & Vídeo & $\begin{array}{l}\text { - Injury Minimization Programme for Schools } \\
\text { - } \text { Con ABC que salva vidas } \\
\text { - PROCES } \\
\text { - ABC for life } \\
\text { - British Heart Foundation Video } \\
\text { - OSNR }\end{array}$ & $\begin{array}{l}\text { - Prevención de accidentes } \\
\text { - SVB } \\
\text { - RCP } \\
\text { DEA } \\
\text { - Respuesta ante las siguientes situaciones: dolor en el } \\
\text { pecho, parada cardíaca, convulsiones severas por } \\
\text { epilepsia, lesiones en la cabeza o el cuello, } \\
\text { quemaduras, hemorragias internas, obstrucción de las } \\
\text { vías aéreas, ahogamiento }\end{array}$ & $\begin{array}{l}\text { (Cerezo Espinosa et al., 2018; } \\
\text { Engeland et al., 2002; Frederick et al., } \\
\text { 2000; Jimenez-Fabrega et al., 2009; } \\
\text { Lester et al., 1996; Lester et al., 1997; } \\
\text { Louis et al., 2015; Onyeaso, 2016; } \\
\text { Palacio et al., 2015; Reder et al., 2006; } \\
\text { Toner et al., 2007; Van Raemdonck et } \\
\text { al., 2014) }\end{array}$ & $\begin{array}{l}\text { Primaria } \\
\text { Secundaria }\end{array}$ \\
\hline \multirow{4}{*}{$\begin{array}{l}\text { Material } \\
\text { informatizado }\end{array}$} & $\begin{array}{l}\text { Plataforma basada en la } \\
\text { Web }\end{array}$ & - Kennisdesk Academy training tool & $\begin{array}{ll}\cdot & \text { SVB } \\
\cdot & \text { RCP } \\
\end{array}$ & (Van Raemdonck et al., 2017) & Secundaria \\
\hline & Programa informático & $\begin{array}{l}\text { - "Protest the Silence" (National Center for } \\
\text { Early Defibrilation, 2003) }\end{array}$ & $\begin{array}{l}\cdot \text { RCP } \\
\text { - } \text { DEA } \\
\end{array}$ & (Reder et al., 2006) & Secundaria \\
\hline & Videojuegos & $\begin{array}{l}\text { - Adventure E-Learning Games (CATEDU, } \\
\text { Aragón) }\end{array}$ & - Teóricos sobre SVB & (Marchiori et al., 2012) & Secundaria \\
\hline & $\begin{array}{l}\text { Tableta informática con } \\
\text { vídeo o imágenes }\end{array}$ & $\cdot$ OSNR & $\begin{array}{ll}\cdot & \text { SVB } \\
\text { - } & \text { RCP }\end{array}$ & (Iserbyt et al., 2014) & Secundaria \\
\hline
\end{tabular}

Nota. DEA = desfibrilación externa automática; OSNR = otros sin nombre registrado; RCP = reanimación cardiopulmonar; SVB = soporte vital básico. 
Younas, Raynes, Morton, \& Mackway-Jones, 2006), y los 4 restantes aplicaron su programa en ambas etapas educativas (Bohn, et al., 2012; Fleischhackl, et al., 2009; Turgut, Yaman, \& Turgut, 2016; Villanueva, Rey, Crespo, Díaz, \& Martínez, 2018).

Los materiales empleados en los diferentes programas formativos fueron muy variados. Entre los materiales didácticos generales (Tabla 2), en el análisis por tipologías, la utilización de materiales de tipo impreso, incorporados en 11 programas formativos (Carruth, et al., 2010; Engeland, et al., 2002; Frederick, et al., 2000; Lester, et al., 1996; López, et al., 2012; Lubrano, et al., 2005; Marchand-Martella, et al., 1992; Palacio, et al., 2015; Toner, et al., 2007; Turgut, et al., 2016; Villanueva, et al., 2018), fue inferior al uso del resto de materiales (i.e. visual fijo o proyectable, auditivo, audiovisual e informatizado), que fueron empleados en 20 de las intervenciones (Bohn, et al., 2012; Cerezo Espinosa, et al., 2018; Engeland, et al., 2002; Frederick, et al., 2000; Garcia del Aguila, et al., 2014; Iserbyt, et al., 2014; Jimenez-Fabrega, et al., 2009; Lester, et al., 1996; Lester, et al., 1997; Louis, et al., 2015; Lubrano, et al., 2005; Marchiori, et al., 2012; Onyeaso, 2016; Palacio, et al., 2015; Reder, et al., 2006; Toner, et al., 2007; Turgut, et al., 2016; Van Raemdonck, et al., 2014; Van Raemdonck, et al., 2017; Villanueva, et al., 2018). Además, en el análisis por elementos materiales, el más utilizado fue el vídeo, integrado en 12 programas (Cerezo Espinosa, et al., 2018; Engeland, et al., 2002; Frederick, et al., 2000; Jimenez-Fabrega, et al., 2009; Lester, et al., 1996; Lester, etal., 1997; Louis, et al., 2015; Onyeaso, 2016; Palacio, et al., 2015; Reder, et al., 2006; Toner, et al., 2007; Van Raemdonck et al., 2014), seguido del manual o libro de texto, incluido en 6 programas (Carruth, et al., 2010; Engeland, et al., 2002; Frederick, et al., 2000; Lubrano, et al., 2005; Marchand-Martella, et al., 1992; Toner, et al., 2007). Otros elementos empleados en menor medida fueron presentaciones de powerpoint, unidades didácticas, fichas de actividades, diploma, carteles, canciones, fichas descriptivas, cuentos, pentadíptico desplegable, insignias, plataforma basada en la Web, programa informático, videojuegos y tableta informática. Por otra parte, entre los materiales didácticos específicos (Tabla 3), en cuanto a su tipología, destacó el uso de material no fungible, móvil, complementario y de medición, que fue incorporado en 19 programas formativos (Bohn, et al., 2012; Fleischhackl, et al., 2009; Garcia del Aguila, et al., 2014;
Iserbyt, et al., 2014; Jimenez-Fabrega, et al., 2009; Kelley, et al., 2006; Lester, et al., 1996; Lester, et al., 1997; López, et al., 2012; Louis, et al., 2015; Meissner, et al., 2012; Onyeaso, 2016; Palacio, et al., 2015; Reder, et al., 2006; Toner, et al., 2007; Van Raemdonck, et al., 2014; Van Raemdonck, et al., 2017; Villanueva, et al., 2018; Younas, et al., 2006), integrándose el resto de tipologías solo en 4 intervenciones (Garcia del Aguila, et al., 2014; Marchand-Martella, et al., 1992; Turgut, et al., 2016; Van Raemdonck, et al., 2014). En el análisis por elementos materiales, ocupó un lugar preeminente el maniquí, integrado en 18 programas (Bohn, et al., 2012; Fleischhackl, et al., 2009; Garcia del Aguila, et al., 2014; Iserbyt, et al., 2014; Jimenez-Fabrega, et al., 2009; Lester, et al., 1996; Lester, et al., 1997; López, et al., 2012; Louis, et al., 2015; Meissner, etal., 2012; Onyeaso, 2016; Palacio, et al., 2015; Reder, et al., 2006; Toner, et al., 2007; Van Raemdonck, et al., 2014; Van Raemdonck, et al., 2017; Villanueva, et al., 2018; Younas, et al., 2006), seguido del desfibrilador externo automático o semiautomático (DEA/DESA), incluido en 7 programas (Fleischhackl, et al., 2009; Kelley, et al., 2006; López, et al., 2012; Meissner, et al., 2012; Reder, et al., 2006; Van Raemdonck, et al., 2017; Younas, et al., 2006). Otros elementos empleados en menor medida fueron teléfono, bolsas de plástico, dados de foam, gasas, alcohol, equipo de simulación de lesiones, equipo de primeros auxilios, animales de peluche y salvavidas.

En cuanto al uso que cada programa realiza de los distintos tipos de materiales (Tablas 2 y 3), si bien ha habido algún programa que emplea materiales de una única clase, la mayoría de los programas optan por una combinación de materiales didácticos generales y específicos, siendo la opción más habitual la mezcla de material audiovisual con material no fungible, móvil, complementario y de medición, integrándose de esta forma 9 de los programas formativos revisados (Jimenez-Fabrega, et al., 2009; Lester, etal., 1996; Lester, et al., 1997; Louis, et al., 2015; Onyeaso, 2016; Palacio, et al., 2015; Reder, et al., 2006; Toner, et al., 2007; Van Raemdonck, et al., 2014). La diversidad de combinaciones es todavía mayor al nivel de los distintos elementos, encontrando toda una gama de composiciones que va desde programas que emplean unúnico elemento material (Carruth, et al., 2010; Kelley, et al., 2006; Marchiori, et al., 2012), a otros que integran hasta cinco (Palacio, et al., 2015; Toner, et al., 2007; Turgut, et al., 2016; Villanueva, et al., 2018), siendo la combina-

Tabla 3

Materiales didácticos específicos empleados en los distintos programas formativos (taxonomía de Fernández, 1999)

\begin{tabular}{|c|c|c|c|c|c|c|c|c|}
\hline \multicolumn{2}{|c|}{ Tipo de material } & Material & Programas & \multirow{2}{*}{\multicolumn{3}{|c|}{ Contenidos }} & Referencias & \multirow[t]{2}{*}{ Etapa educativa } \\
\hline \multirow{2}{*}{\multicolumn{9}{|c|}{ Alternativo (o No convencional) }} \\
\hline & & & & & & & & \\
\hline \multirow{4}{*}{ Económico } & Bolsa de plástico & & - OSNR & $\begin{array}{l}\cdot \text { SVB } \\
\cdot \quad \text { RCP } \\
\end{array}$ & & & (Van Raemdonck et al., 2014) & Secundaria \\
\hline & Dado de foam & & - OSNR & $\begin{array}{l}\cdot \text { SVB } \\
\cdot \quad \text { RCP } \\
\end{array}$ & & & (Van Raemdonck et al., 2014) & Secundaria \\
\hline & Gasas & & $\begin{array}{l}\text { - Cardiomaratón } \\
\text { (formación masiva) }\end{array}$ & $\begin{array}{ll} & \text { Rec } \\
& \text { RCP } \\
\text { - } & \text { PLS } \\
\text { - } & \text { Cade } \\
\text { - } & \text { Llan } \\
\end{array}$ & $\begin{array}{l}\text { onocer PCR } \\
\text { b } \\
\text { ena de supervivencia } \\
\text { nada s centro coordinador de urgenc } \\
\text { gantamiento y desobstrucción de vía }\end{array}$ & ias & (Garcia del Aguila et al., 2014) & Secundaria \\
\hline & Alcohol & & $\begin{array}{l}\text { - Cardiomaratón } \\
\text { (formación masiva) }\end{array}$ & 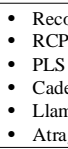 & $\begin{array}{l}\text { nocer PCR } \\
\text { b } \\
\text { ena de supervivencia } \\
\text { ada a centro coordinador de urgenc } \\
\text { gantamiento y desobstrucción de vía }\end{array}$ & $\begin{array}{l}\text { iias } \\
\text { a aérea }\end{array}$ & (Garcia del Aguila et al., 2014) & Secundaria \\
\hline \multirow{3}{*}{ Comercial } & $\begin{array}{l}\text { Equipo de simulación } \\
\text { lesiones, cera de emb }\end{array}$ & $\begin{array}{l}\text { le lesiones (parches de } \\
\text { samar, sangre simulada, etc.) }\end{array}$ & - Mouse Calls & - Tratc & amiento de: abrasiones, quemaduras & y cortes severos & (Marchand-Martella et al., 1992) & Primaria \\
\hline & Equipo de primeros a & ilios & - Mouse Calls & - Trate & amiento de: abrasiones, quemaduras & y cortes severos & (Marchand-Martella et al., 1992) & Primaria \\
\hline & Animales de peluche & & - Mouse Calls & - Trata & amiento de: abrasiones, quemaduras & y cortes severos & (Marchand-Martella et al., 1992) & Primaria \\
\hline \multicolumn{9}{|c|}{ Materiales No Fungibles } \\
\hline \multicolumn{9}{|l|}{ Móvil } \\
\hline \multirow[t]{2}{*}{$\begin{array}{l}\text { Convencional (o } \\
\text { reglamentario) }\end{array}$} & Línea salvavidas & - OSNR & & & - Seguridad en el agua & \multicolumn{2}{|c|}{ (Turgut et al., 2016) } & $\begin{array}{l}\text { Primaria } \\
\text { Secundaria }\end{array}$ \\
\hline & DEA / DESA & $\begin{array}{ll}\text { - } & \text { Kennisdesk Academy trai } \\
\text { - Opportunities for Resusci } \\
\text {. } & \text { OSP na aula } \\
\end{array}$ & $\begin{array}{l}\text { ining tool } \\
\text { tation and Citizen Safety }\end{array}$ & (ORCS) & $\begin{array}{ll}\cdot & \text { SVB } \\
\text { - } & \text { RCP } \\
\text { - } & \text { DEA/DESA } \\
\end{array}$ & $\begin{array}{l}\text { (Fleischhackl et a } \\
\text { et al., 2012; Meis } \\
\text { Van Raemdonck }\end{array}$ & $\begin{array}{l}\text { l., 2009; Kelley et al., 2006; López } \\
\text { ssner et al., 2012; Reder et al., 2006; } \\
\text { et al., 2017; Younas et al., 2006) }\end{array}$ & $\begin{array}{l}\text { Primaria } \\
\text { Secundaria }\end{array}$ \\
\hline \multirow[t]{2}{*}{$\begin{array}{l}\text { Complementario } \\
\text { y de medición }\end{array}$} & Maniquí & $\begin{array}{ll}\text { - } & \text { Kennisdesk Academy trai } \\
\text { - Oportunities for Resusci } \\
\text { PROCES } \\
\text { - RCP na aula } \\
\text { - Eardiomaratón (formació } \\
\text { : } & \text { ABC for life } \\
\text { - OSNR }\end{array}$ & $\begin{array}{l}\text { Ining tool } \\
\text { tation and Citizen Safety } \\
\text { n masiva) }\end{array}$ & (ORCS) & $\begin{array}{ll} & \text { SVB } \\
\text { - } & \text { RCP } \\
\text { DEA/DESA } \\
\text { - } & \text { Reconocer PCR } \\
\text { PLS } \\
\text { - } \text { Cadena de supervivencia } \\
\text { de urgada a centro coordinador } \\
\text { - } \\
\text { Atragantamiento y } \\
\text { desobstrucción de vía aérea. }\end{array}$ & \multicolumn{2}{|c|}{$\begin{array}{l}\text { (Bohn et al., 2012; Fleischhackl et al., 2009; Garcia } \\
\text { del Aguila et al., 2014; Iserbyt et al., 2014; Jimenez- } \\
\text { Fabrega et al., 2009; Lester et al., 1996; Lester et al., } \\
\text { 1997; López et al., 2012; Louis et al., 2015; Meissner } \\
\text { et al., 2012; Onyeaso, 2016; Palacio et al., 2015; } \\
\text { Reder et al., 2006; Toner et al., 2007; Van } \\
\text { Raemdonck et al., 2014; Van Raemdonck et al., 2017; } \\
\text { Villanueva et al., 2018; Younas et al., 2006) }\end{array}$} & $\begin{array}{l}\text { Primaria } \\
\text { Secundaria }\end{array}$ \\
\hline & Teléfono & $\begin{array}{l}\text { - Opportunities for Resusci } \\
\text { - OSNR }\end{array}$ & tation and Citizen Safety & (ORCS) & $\begin{array}{l}\cdot \text { SVB } \\
\cdot \text { RCP } \\
\end{array}$ & $\begin{array}{l}\text { (Fleischhackl et a } \\
\text { Younas et al., } 200\end{array}$ & $\begin{array}{l}\text { l., 2009; Villanueva et al., 2018; } \\
\text { 06) }\end{array}$ & $\begin{array}{l}\text { Primaria } \\
\text { Secundaria }\end{array}$ \\
\hline
\end{tabular}


ción más repetida la del vídeo, para la transmisión de contenidos teóricos o teórico-prácticos, con el maniquí, empleado predominantemente para la enseñanza de las habilidades prácticas requeridas para la reanimación cardiopulmonar y para evaluar la adquisición de las mismas.

Por último, algunos estudios han analizado, de forma específica, la eficacia obtenida en distintos programas formativos en función del material utilizado, esto es, si cabe atribuir una mayor eficacia o calidad formativa a unos materiales que a otros. Entre ellos, no se encontraron diferencias en la eficacia formativa de un programa para la transmisión de los contenidos teóricos relativos al soporte vital básico (SVB), en función de si el diseño del mismo comprendía formación cara a cara con soporte de presentaciones en powerpoint, o de si se presentaban los contenidos a través de un polimedia (composición de medios audiovisuales) (Cerezo Espinosa, et al., 2018). Por su parte, Louis et al. (2015) apreciaron que los contenidos en línea presentados en formato de vídeo favorecen la retención y la revisión de conocimientos requeridos para la RCP, mientras que la formación práctica con utilización de maniquí y desfibrilador externo automático (DEA) mejora la confianza y el compromiso para difundir dichos conocimientos en las escuelas. En otro estudio se apreció que la autoinstrucción por medio de un programa informático interactivo resulta suficiente para enseñar los conceptos relacionados con la RCP y la desfibrilación externa automática, así como para las acciones correspondientes a esta última, sin embargo, el nivel de aprendizaje logrado en cuanto a las habilidades requeridas para la RCP fue insuficiente (Reder, et al., 2006). Desde otra perspectiva, se apuntó que los fundamentos de la RCP pueden ser enseñados con material alternativo si no se dispone de maniquíes, al observar que la eficacia de la formación con materiales de bajo coste no difirió de la lograda con el uso del maniquí, si bien en ambos casos los resultados fueron subóptimos (Van Raemdonck, et al., 2014). También se obtuvieron resultados razonablemente buenos en la transmisión de contenidos teóricos relativos al SVB por medio de un videojuego, si bien el programa no alcanzó los niveles de rendimiento que se logran con una formación más reglada, basada en instructor acreditado en RCP (Marchiori, et al., 2012). Por último, al comparar la eficacia lograda mediante el empleo de imágenes frente al vídeo, ambos soportados en una tableta informática, a la hora de formar en RCP, no se apreciaron diferencias entre los dos grupos en la profundidad de las compresiones, pero la colocación de las manos fue mejor en el grupo formado mediante imágenes (Iserbyt, et al., 2014).

\section{Discusión}

Existe constancia de un elevado número de programas de enseñanza de primeros auxilios que han sido o están siendo aplicados en las distintas etapas que componen la escolaridad obligatoria. En el diseño de estos planes formativos, la disposición y configuración de los materiales representa un aspecto clave y se aprecia una enorme variedad de combinaciones, a menudo sin proporcionar una justificación para la opción escogida.

Entre los programas que se han mostrado eficaces, se debe tener en cuenta que la investigación respalda la utilización de material audiovisual o informatizado para la transmisión de contenidos eminentemente teóricos o conceptuales (Cerezo Espinosa, et al., 2018; Marchiori, et al., 2012), pero que la adquisición de algunas habilidades o destrezas, como las necesarias para aplicar correctamente la RCP, requiere a menudo el diseño de programas con un componente práctico (Louis, et al., 2015; Reder, et al., 2006). Asimismo, resulta común el uso de una combinación de materiales didácticos generales y específicos, siendo la opción más habitual la mezcla de material impreso o audiovisual con material no fungible, móvil, complementario y de medición, esto es, manual certificado de primeros auxilios, vídeo, maniquí y DEA (Jimenez-Fabrega, et al., 2009; Lester, et al., 1996; Lester, et al., 1997; Louis, et al., 2015; Onyeaso, 2016; Palacio, et al., 2015; Reder, et al., 2006; Toner, et al., 2007; Van Raemdonck, et al., 2014), por lo que cabe recomendar el empleo de una combinación similar a la hora de diseñar futuros programas formativos, siempre y cuando se disponga de dichos materiales o de los medios económicos oportunos para adquirirlos. Desde el punto de vista de la optimización de los recursos, resultan interesantes las iniciativas formativas en las que, mediante la colaboración entre instituciones o administraciones (como las de salud y las de educación), pueden compartirse algunos de los materiales más costosos, como el maniquí y el DEA (Louis, et al., 2015). No obstante, la carencia de estos materiales no debería representar un obstáculo insalvable para la aplicación de programas de enseñanza eficaces ya que, tal como se ha constatado (Van Raemdonck, et al., 2014), existen materiales específicos, fungibles, alternativos, económicos, como una bolsa de plástico y un dado de foam, que pueden ser empleados con éxito en su lugar. Otras posibilidades, todavía sin explorar en la investigación, dentro de los materiales específicos, fungibles, vendrían constituidas por el material deportivo (e.g. pelotas y balones) o de psicomotricidad(e.g. globos) que también podrían ser utilizados, de forma adaptada, para sustituir al maniquí, y que resultan especialmente recomendables para los maestros de educación física, por constituir materiales generalmente disponibles en todos los centros educativos.

Entre las limitaciones apreciadas en la investigación recopilada, cabe señalar que a menudo se observa una información deficitaria en cuanto a los materiales empleados en los programas formativos, lo que impide determinar con precisión sus características y uso, obstaculizando, en consecuencia, tanto la replicación de las intervenciones como la evaluación adecuada de la eficacia de los programas, ya que se dificulta la atribución de los resultados a los aspectos relativos a los materiales didácticos o a algún otro factor, así como la comparación con otros programas de enseñanza. A este respecto, resulta relevante no solo la calidad y características de los materiales, sino también su cantidad, ya que la ratio que se establece entre los distintos elementos materiales y el número de alumnos puede resultar un indicador especialmente importante en los programas con componente práctico puesto que las ratios bajas conllevan tiempo de inactividad en el alumnado y, por tanto, un mal aprovechamiento del tiempo y pocas oportunidades de práctica. Asimismo, resultaría necesaria la justificación de la combinación de materiales escogidos, para una mejor comprensión de si esta se basa en criterios didácticos o cualquier otro tipo de razones. En futuras investigaciones o planes formativos, podría tener cabida la utilización de tipologías de material didáctico todavía por explorar, como las mencionadas en el párrafo anterior, o las que constituyen los materiales específicos, fungibles, alternativos, de desecho y autofabricados (e.g. sacos rellenos de trapos o ropa para sustituir a los maniquíes, simuladores de DEA construidos con cajas, córdeles y algún adhesivo, etc.).

Por último, en cuanto a las posibilidades del maestro de educación física de ejercer como instructor de primeros auxilios, en investigaciones anteriores se ha reportado que el hecho de que dicho papel sea desempeñado por un médico de emergencias, comparado con la labor desarrollada por maestros, no condujo a un mayor aprendizaje de la RCP (Bohn, et al., 2012). Asimismo, los resultados previos de investigación soportan a los profesores de educación física debidamente certificados en primeros auxilios y RCP como instructores adecuados para la formación de los escolares (Reveruzzi, Buckley, \& Sheehan, 2016). Desde el punto de vista de la elección, creación y disposición de los materiales didácticos y el diseño de los programas formativos, parece lógico pensar que, como especialistas que son en los procesos de enseñanzaaprendizaje en las edades escolares, los maestros representan el complemento profesional ideal a los especialistas sanitarios para lograr, con la mayor eficacia posible, la transmisión de los conocimientos y habilidades involucradas en los primeros auxilios.

\section{Conclusiones}

Existe una enorme variedad de materiales a disposición de los maestros en educación física para llevar a cabo la enseñanza de los primeros auxilios. Ala hora de diseñar un programa formativo en primeros auxilios, deberían emplearse criterios didácticos para seleccionar y combinar los materiales a emplear. A la luz de la evidencia disponible, resulta recomendable el empleo de una combinación de materiales didácticos generales y específicos, siendo la mejor opción una mezcla de material 
audiovisual o impreso, para los contenidos teóricos, con material no fungible, móvil, complementario y de medición, para la formación práctica, esto es, al menos debería disponerse de manual certificado de primeros auxilios, vídeo, maniquí y DEA. En caso de no disponer de maniquí y DEA, existen alternativas de bajo coste que proporcionan un nivel de eficacia similar.

\section{Referencias}

American Heart Association. (2001). Heartsaver CPR in the schools: Student manual. Dallas: American Heart Association National Center.

Bohn, A., Van Aken, H. K., Mollhoff, T., Wienzek, H., Kimmeyer, P., Wild, E., ... Weber, T. P. (2012). Teaching resuscitation in schools: Annual tuition by trained teachers is effective starting at age 10. A four-year prospective cohort study. Resuscitation, 83(5), 619-625. doi:10.1016/j.resuscitation.2012.01.020

Böttiger, B. W., Bossaert, L. L., Castrén, M., Cimpoesu, D., Georgiou, M., Greif, R.,...Melieste, R. (2016). Kids Save Lives-ERC position statement on school children education in CPR: "Hands that helpTraining children is training for life”. Resuscitation, 105, A1-A3. doi:10.1016/j.resuscitation.2016.06.005.

Carruth, A. K., Pryor, S., Cormier, C., Bateman, A., Matzke, B., \& Gilmore, K. (2010). Evaluation of a school-based train-the-trainer intervention program to teach first aid and risk reduction among high school students. The Journal of School Health, 80(9), 453460. doi:10.1111/j.1746-1561.2010.00527.x

Cerezo Espinosa, C., Nieto Caballero, S., Juguera Rodriguez, L., Castejon-Mochon, J. F., Segura Melgarejo, F., Sanchez Martinez, C. M., . . . Pardo Rios, M. (2018). Learning cardiopulmonary resuscitation theory with face-to-face versus audiovisual instruction for secondary school students: A randomized controlled trial. [Ensayo clinico aleatorizado controlado que compara la formacion presencial frente a la no presencial en el aprendizaje teorico de la reanimacion cardiopulmonar entre los estudiantes de secundaria] Emergencias: Revista De La Sociedad Espanola De Medicina De Emergencias, 30(1), 28-34.

Engeland, A., Roysamb, E., Smedslund, G., \& Sogaard, A. J. (2002). Effects of first-aid training in junior high schools. Injury Control and Safety Promotion, 9(2), 99-106. doi:10.1076/icsp.9.2.99.8702

Fernández, J. C. (1999). Aplicación didáctica de los recursos materiales en el diseño curricular de educación física. Sevilla: Fondo Editorial Fundación San Pablo C.E.U.

Fleischhackl, R., Nuernberger, A., Sterz, F., Schoenberg, C., Urso, T., Habart, T., . . . Chandra-Strobos, N. (2009). School children sufficiently apply life supporting first aid: A prospective investigation. Critical Care (London, England), 13(4), R127. doi:10.1186/cc7984

Frederick, K., Bixby, E., Orzel, M. N., Stewart-Brown, S., \& Willett, K. (2000). An evaluation of the effectiveness of the injury minimization programme for schools (IMPS). Injury Prevention: Journal of the International Society for Child and Adolescent Injury Prevention, 6(2), 92-95.

Garcia del Aguila, J. J., Lopez-Rebollo, E., Garcia-Sanchez, M., VivarDiaz, I., Alba Resina, A., Fierro-Roson, F. J., . . Rosell-Ortiz, F. (2014). Mass training in basic life support for high-school students. Resuscitation, 85(5), e67-8. doi:10.1016/j.resuscitation.2014.01.001

García-Vega, F. J., Montero-Pérez, F. J., \& Encinas-Puente, R. M. (2008). La comunidad escolar como objetivo de la formación en resucitación. la RCP en las escuelas. Emergencias, 20(4), 223-225.

Iserbyt, P., Charlier, N., \& Mols, L. (2014). Learning basic life support (BLS) with tablet PCs in reciprocal learning at school: Are videos superior to pictures? A randomized controlled trial. Resuscitation, 85(6), 809-813. doi:10.1016/j.resuscitation.2014.01.018

Jelinek, G. A., Gennat, H., Celenza, T., O’Brien, D., Jacobs, I., \& Lynch, D. (2001). Community attitudes towards performing cardiopulmonary resuscitation in western australia. Resuscitation,

\section{1(3), 239-246. doi:S0300957201004117}

Jimenez-Fabrega, X., Escalada-Roig, X., Miro, O., Sanclemente, G., Diaz, N., Gomez, X., ... Sanchez, M. (2009). Comparison between exclusively school teacher-based and mixed school teacher and healthcare provider-based programme on basic cardiopulmonary resuscitation for secondary schools. Emergency Medicine Journal: EMJ, 26(9), 648-652. doi:10.1136/emj.2008.062992

Jones, I., Whitfield, R., Colquhoun, M., Chamberlain, D., Vetter, N., \& Newcombe, R. (2007). At what age can schoolchildren provide effective chest compressions? an observational study from the heartstart UK schools training programme. BMJ(Clinical Research Ed.), 334(7605), 1201. doi:bmj.39167.459028.DE

Kelley, J., Richman, P. B., Ewy, G. A., Clark, L., Bulloch, B., \& Bobrow, B. J. (2006). Eighth grade students become proficient at CPR and use of an AED following a condensed training programme. Resuscitation, 71(2), 229-236. doi:S0300-9572(06)00152-3

Lester, C., Donnelly, P., \& Weston, C. (1997). Is peer tutoring beneficial in the context of school resuscitation training? Health Education Research, 12(3), 347-354.

Lester, C., Donnelly, P., Weston, C., \& Morgan, M. (1996). Teaching schoolchildren cardiopulmonary resuscitation. Resuscitation, 31(1), 33-38. doi:0300957295009124

Liberman, M., Golberg, N., Mulder, D., \& Sampalis, J. (2000). Teaching cardiopulmonary resuscitation to CEGEP students in quebec - a pilot project. Resuscitation, 47(3), 249-257. doi:S0300957200002367

López, M. C., Freire, M., Rasines, R., \& Iglesias, A. (2012). RCP na aula: Programa de enseñanza de la reanimación cardiopulmonar básica para estudiantes de secundaria. Emergencias, 24, 74-80.

López-Felpeto, R., Navarro-Patón, R., \& Basanta-Camiño, S. (2015). Formación y actitud del profesorado de educación física en educación primaria con respecto a los primeros auxilios en la provincia de lugo. Trances, 7(1), 91-112.

Louis, C. J., Reyero, D., Beumont, C., Beramendi, F., Roldan, J., \& Fernandez, A. (2015). Design thinking tools empower teachers to spread CPR training in school. the el ABC que salva vidas program. Resuscitation, 96(1), 84-85.

Lubrano, R., Romero, S., Scoppi, P., Cocchi, G., Baroncini, S., Elli, M., ... Moscatelli, R. (2005). How to become an under 11 rescuer: A practical method to teach first aid to primary schoolchildren. Resuscitation, 64(3), 303-307. doi:S0300-9572(04)00379-X

Marchand-Martella, N. E., Martella, R. C., Christensen, A. M., Agran, M., \& Young, K. R. (1992). Assessing the duration of first-aid treatments by elementary-aged students with disabilities. Child \& Family Behavior Therapy, 14(3), 33-52. doi:http://dx.doi.org/ 10.1300/J019v14n03_03

Marchiori, E. J., Ferrer, G, Fernández-Manjón, B., Povar-Marco, J., Suberviola, J. F., \& Giménez-Valverde, A. (2012). Instrucción en maniobras de soporte vital básico mediante videojuegos: Comparación de resultados frente a un grupo control. Emergencias, 24, 433437.

Meissner, T. M., Kloppe, C., \& Hanefeld, C. (2012). Basic life support skills of high school students before and after cardiopulmonary resuscitation training: A longitudinal investigation. Scandinavian Journal of Trauma, Resuscitation and Emergency Medicine, 20, 31-7241-20-31. doi:10.1186/1757-7241-20-31 [doi]

Miró, O., Díaz, N., Escalada, X., Pérez-Pueyo, F., \& Sánchez, M. (2012). Revisión de las iniciativas llevadas a cabo en españa para implementar la enseñanza de la reanimación cardiopulmonar básica en las escuelas. Anales Del Sistema Sanitario De Navarra, 35(3), 477-486.

Miró, O., Díaz, N., Escalada, X., Pérez-Pueyo, F., \& Sánchez, M. (2013). Puntos clave para introducir la enseñanza de la reanimación cardiopulmonar básica en las escuelas. Salud i Ciencia, 20(3), 251256.

Mpotos, N., Vekeman, E., Monsieurs, K., Derese, A., \& Valcke, M. (2013). Knowledge and willingness to teach cardiopulmonary 
resuscitation: Asurvey amongst 4273 teachers. Resuscitation, 84(4), 496-500. doi:10.1016/j.resuscitation.2013.01.023

Navarro, R., Arufe, V., \& Basanta, S. (2015). Estudio descriptivo sobre la enseñanza de los primeros auxilios por el profesorado de educación física en centros de educación primaria. Sportis. Revista Técnico-Científica Del Deporte Escolar, Educación Física Y Psicomotricidad, 1(1), 35-52.

Navarro-Patón, R., Basanta-Camiño, S., Abelairas-Gómez, C., \& LópezGarcía, S. (2015). Análisis de la situación de los primeros auxilios en los planes de estudio de los grados de maestra y maestro de educación primaria. Trances, 7(4), 599-612.

Navarro-Patón, R., Freire-Tellado, M., Basanta-Camiño, S., BarcalaFurelos, R.,Arufe-Giráldez, V., \& Rodríguez-Fernández, J. E. (2018). Efecto de 3 métodos de enseñanza en soporte vital básico en futuros maestros de educación primaria. un diseño cuasi-experimental. Medicina Intensiva, 42(4), 207-215. doi:10.1016/ j.medin.2017.06.005

Navarro-Patón, R., Penelas-Teijeiro, G., \& Basanta-Camiño, S. (2016). ¿Tienen las futuras maestras y maestros de educación primaria la formación necesaria para iniciar las maniobras de reanimación cardiopulmonar en caso de emergencia escolar? un estudio descriptivo. Educar, 52(1), 149-168. doi:10.5565/rev/educar.714

Nolan, J. P., Soar, J., Zideman, D. A., Biarent, D., Bossaert, L. L., Deakin, C., . . ERC Guidelines Writing Group. (2010). European resuscitation council guidelines for resuscitation 2010 section 1. executive summary. Resuscitation, 81(10), 1219-1276. doi:10.1016/ j.resuscitation.2010.08.021

Onyeaso, A. O. (2016). Retention of cardiopulmonary resuscitation skills in nigerian secondary school students. Journal of Education and Practice, 7(15), 162-168.

Palacio, R., Nonide, M., Carreño, F., López, L., \& Cao, A. (2015). Proyecto «con tus manos puedes salvar vidas». RqR Enfermería Comunitaria (Revista De SEAPA), 3(2), 35-43.

Real Decreto 126/2014, de 28 de febrero, por el que se establece el currículo básico de la Educación Primaria. Boletín Oficial del Estado, 52, 19349-19420. Recuperado de: http://www.boe.es/boe/ dias/2014/03/01/pdfs/BOE-A-2014-2222.pdf

Real Decreto 1105/2014, de 26 de diciembre, por el que se establece el currículo básico de la Educación Secundaria Obligatoriay del Bachillerato. Boletín Oficial del Estado, 3, 169-546. Recuperado de: http:/ /www.boe.es/boe/dias/2015/01/03/pdfs/BOE-A-2015-37.pdf

Reder, S., Cummings, P., \& Quan, L. (2006). Comparison of three instructional methods for teaching cardiopulmonary resuscitation and use of an automatic external defibrillator to high school students.
Resuscitation, 69(3), 443-453. doi:S0300-9572(05)00444-2

Reder, S., \& Quan, L. (2003). Cardiopulmonary resuscitation training in washington state public high schools. Resuscitation, 56(3), 283288. doi:S0300957202003763 [pii]

Reveruzzi, B., Buckley, L., \& Sheehan, M. (2016). School-based first aid training programs: A systematic review. The Journal of School Health, 86(4), 266-272. doi:10.1111/josh.12373 [doi]

Ríos, R. (2011). Efectividad de un programa formativo «pensando y actuando» en los conocimientos de los docentes de niños especiales sobre primeros auxilios en el centro de educación básica especial reverenda madre mariana carrigan, san juan de miraflores. Unpublished Facultad de Medicina Humana, Lima, Perú.

Teijeiro-Blanco, L., Navarro-Patón, R., \& Basanta-Camiño, S. (2017). La educación para la salud en la didáctica de la educación física en educación primaria. efecto de un programa audiovisual de primeros auxilios en las clases de educación fisica. Trances, 9(2), 113-130.

Toner, P., Connolly, M., Laverty, L., McGrath, P., Connolly, D., \& McCluskey, D. R. (2007). Teaching basic life support to school children using medical students and teachers in a 'peer-training' model - results of the 'ABC for life' programme. Resuscitation, 75(1), 169-175. doi:S0300-9572(07)00169-4

Turgut, T., Yaman, M., \& Turgut, A. (2016). Educating children on water safety for drowning prevention. Social Indicators Research, 129(2), 787-801. doi:http://dx.doi.org/10.1007/s11205-015-11090

Van Raemdonck, V., Monsieurs, K. G., Aerenhouts, D., \& De Martelaer, K. (2014). Teaching basic life support: A prospective randomized study on low-cost training strategies in secondary schools. European Journal of Emergency Medicine: Official Journal of the European Society for Emergency Medicine, 21(4), 284-290. doi:10.1097/ MEJ.0000000000000071

Van Raemdonck, V., Aerenhouts, D., Monsieurs, K., \& De Martelaer, K. (2017). A pilot study of flipped cardiopulmonary resuscitation training: Which items can be self-trained? Health Education Journal, 76(8), 946-955.

Villanueva, M. J., Rey, C., Crespo, F., Díaz, L., \& Martínez, G. (2018). Análisis de una experiencia perdurable de un proyecto educativo de reanimación cardiopulmonar (RCP) en un centro escolar. Emergencias, In Press

Younas, S., Raynes, A., Morton, S., \& Mackway-Jones, K. (2006). An evaluation of the effectiveness of the opportunities for resuscitation and citizen safety (ORCS) defibrillator training programme designed for older school children. Resuscitation, 71(2), 222-228. doi:S03009572(06)00149-3
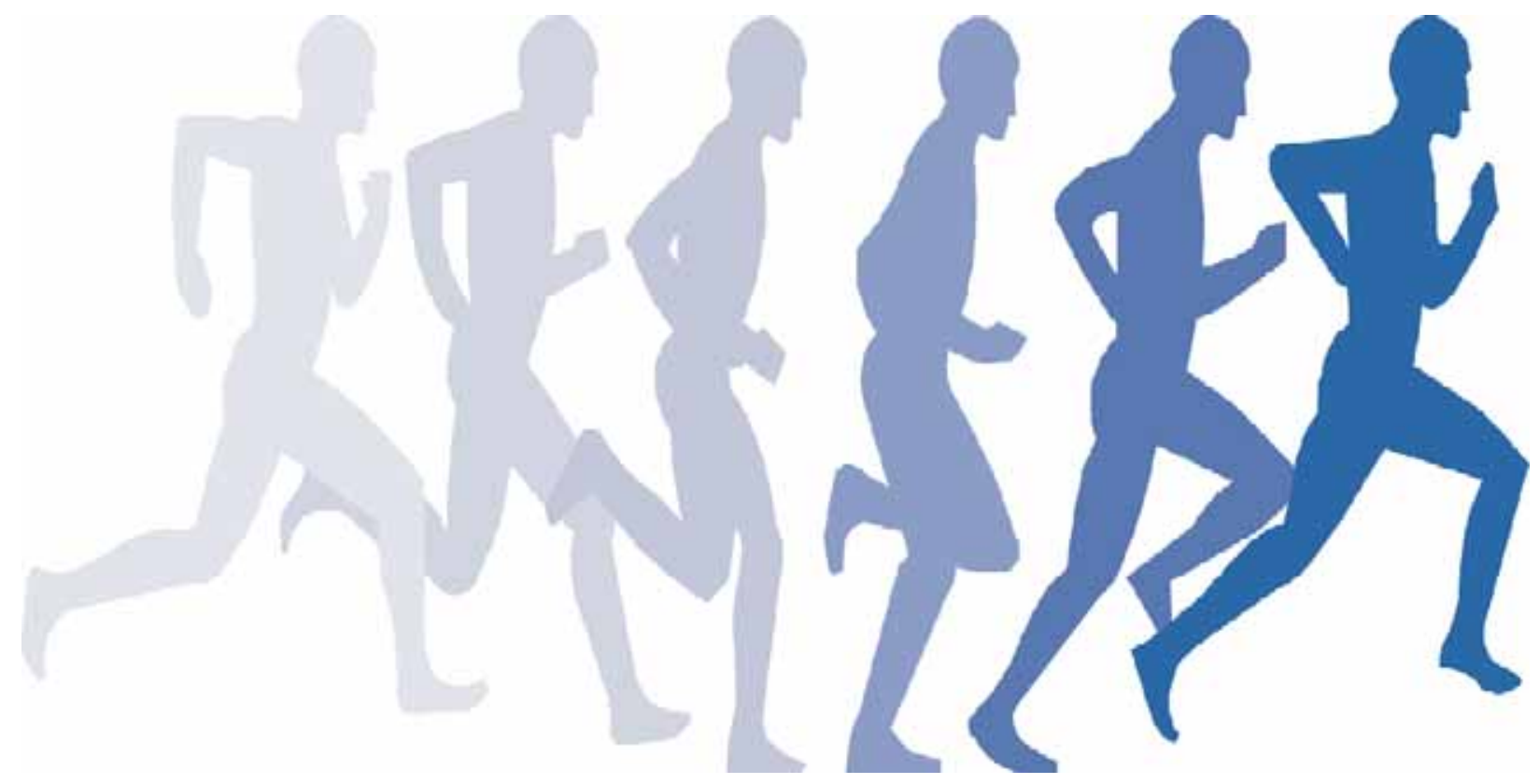